\title{
Emulation of $\pi$-Electron Systems with Mechanical Waves: Borazine
}

\author{
R.A. Méndez-SÁnchez ${ }^{a, *}$, A.E. Terán-Juárez ${ }^{a}$, \\ A.M. Martínez-Argǘlllo ${ }^{a}$, E. Flores-Olmedo ${ }^{b}$, \\ G. BÁEZ ${ }^{b}$ E. SAdurní $i^{c}$ And Y. Hernández-EsPinosa ${ }^{d}$
}

${ }^{a}$ Instituto de Ciencias Físicas, Universidad Nacional Autónoma de México, P.O. Box 48-3, 62251 Cuernavaca, Mor. Mexico

${ }^{b}$ Departamento de Ciencias Básicas, Universidad Autònoma Metropolitana-Azcapotzalco, Av. San Pablo 180, Col. Reynosa Tamaulipas, 02200, Ciudad de México, Mexico

${ }^{c}$ Benemérita Universidad Autónoma de Puebla, Instituto de Física, Apartado Postal J-48, 72570 Puebla, Mexico

${ }^{d}$ Universidad Nacional Autónoma de México, Instituto de Física, Apartado Postal 20-364, CDMX 01000, Mexico

Doi: 10.12693/APhysPolA.140.532

*e-mail: mendez@icf.unam.mx

\begin{abstract}
Elastic waves have emerged as a paradigm of condensed matter physics since many dynamical effects of the latter can be emulated with these waves. Molecular $\pi$-orbitals are of great interest in this area because they play a key role in the electronic transport of $2 \mathrm{D}$ materials and aromatic molecules such as benzene and borazine. In this work, we show the design, construction and characterization of an elastic, artificial borazine structure that emulates the $\pi$-orbitals of borazine. The elastic structure consists of hexagonal resonators that act as artificial elastic atoms coupled to each other through finite phononic crystals that in turn act as artificial $\pi$ bonds. When the resonant frequency of an artificial atom falls within the gap of the phononic crystal, the vibrations are trapped in the artificial atom and interact weakly with neighboring artificial atoms through evanescent Bloch waves. Thus, a tight-binding regime for elastic waves emerges. Here, these ideas are applied to emulate the $\pi$-orbitals of borazine. The design relies on extensive finite element numerical simulations. The experimental results show an excellent agreement with both, the tight-binding formalism and the numerical results.
\end{abstract}

topics: aromatic molecules, finite groups, atomic orbitals, tight-binding models

\section{Introduction}

Elastic waves have emerged as a paradigm in condensed matter physics as several phenomena occurring in this field can be found in elastic wave systems in a new field known as elastic or phononic metamaterials. Topologically protected edge states [1, 2], artificial graphene [3, 4], mechanical negative refraction [5], and other effects appear in elastic waves. In fact, it has been recently demonstrated that it is also possible to emulate molecular $\pi$ systems with elastic waves and thus a tight-binding (TB) regime for elastic waves emerges [6-9]. Thus an alternative to microwave systems with dielectric resonators [10] has been found in elastic waves.

In the TB regime for elastic waves, coupledresonator elastic (or phononic) metamaterials (CREMs) emerge. These metamaterials are inspired by the coupled-resonant optical waveguides [11, 12] that gave rise to the coupled-resonator elastic waveguides $[6-8,13-15]$. In contradistinction to these works, the CREMs [6-9] is used to emulate quantum systems and not to guide acoustic waves. In elastic waves, the spectrum and orbitals of artificial elastic ethylene [6], hexatriene [7], and those of the artificial elastic benzene [9] have been measured. Also, a theoretical framework for 1D systems has been developed [8]. In the CREMs the elastic resonators are coupled to each other through finite phononic crystals (FPC) also known as locally periodic elastic systems [16-22]. When the normalmode frequency of the resonator falls within a forbidden band of the FPC, the normal-mode wave amplitudes localize in the resonators due to the elastic Bragg reflection and the TB regime for elastic waves emerges.

Borazine, also known as inorganic benzene, is a ring-shaped plane molecule composed of three nitrogen atoms interspersed by three boron atoms and six hydrogen atoms at the border (see Fig. 1). 


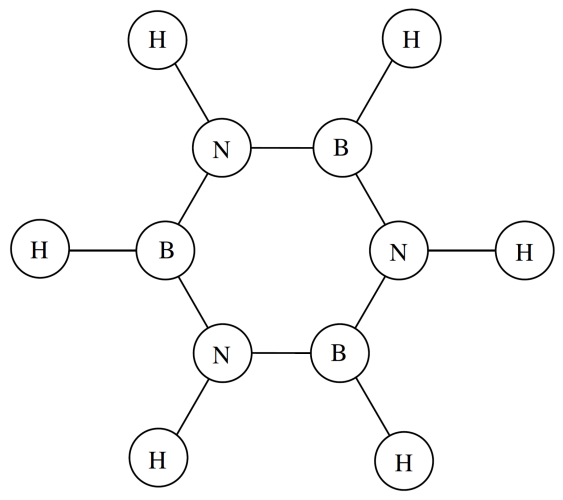

Fig. 1. Schematic diagram of borazine. It is composed by three boron atoms (B), three nitrogen atoms $(\mathrm{N})$ and six hydrogen atoms $(\mathrm{H})$.

In borazine, partial localization and moderate aromaticity has been found by DFT and using group theory with the TB model [23, 24]. In this paper, the elastic borazine is studied numerically and experimentally, using finite elements and the single frequency resonant ultrasound spectroscopy, respectively.

In Sect. 2, the elastic borazine is presented. Its frequency spectrum is calculated numerically using finite elements; this is done in Sect. 3. In Sect. 4 the tight-binding model for the elastic borazine is given. In Sect. 5 experiments based on a variation of the resonant ultrasound spectroscopy technique are presented. They show good agreement with the numerical simulations and with the results of the TB model. As it will be seen in the conclusions, the spectrum and symmetries of the orbitals of the elastic borazine agree with those obtained from the quantum tight-binding model and show partial localization.

\section{The elastic analog of borazine}

The first ingredient to build the elastic analog of borazine is a set of six hexagonal resonators, three $\left(R_{1}, R_{3}\right.$, and $\left.R_{5}\right)$ of length $L_{1}$ and three $\left(R_{2}, R_{4}\right.$, and $\left.R_{6}\right)$ of length $L_{2}$. Each resonator is a thin hexagonal plate of thickness $h$. The frequency spectrum of a hexagonal plate, as a function of the hexagon length $L_{h}$, calculated numerically using the finite element method (COMSOL Multiphysics), with free boundary conditions, is given in Fig. 2a. As expected, the frequencies increase as the hexagon length decreases. The normal-mode wave amplitudes of the modes with lowest frequencies are given in Fig. 2b. As it can be observed in Fig. 2, the two lowest modes of the hexagon are degenerate. The third mode only has an annular nodal line whereas the fourth mode has radial nodes that hit the center of each hexagon length. The latter mode will be used as an artificial atomic orbital. Finite phononic crystals [16, 17, 20-22], also known as locally periodic systems $[18,19]$, are the second ingredient needed to build the elastic artificial borazine. The finite phononic crystals used are built repeating a $2 \mathrm{D}$ unit cell in one dimension. For borazine, each unit cell is composed of a large square plate of length $L$ and two small cuboids of $b \times a / 2 \times h$. A finite phononic crystal of nine unit cells is given in Fig. 3a whose spectrum, as a function of the side length of the square, is given in Fig. 2c. As can be seen, the spectrum shows bands and gaps. When a normal-mode frequency of a resonator falls within a bandgap of the phononic crystal, the normal mode is trapped due to elastic or phononic Bragg reflection [25, 26]. This is accomplished by changing the length of the resonator. In Fig. 3c, a localized wave amplitude on a meta-atom is given. This localized wave amplitude will be called artificial elastic $\pi$-orbital, or elastic meta-orbital. (a)

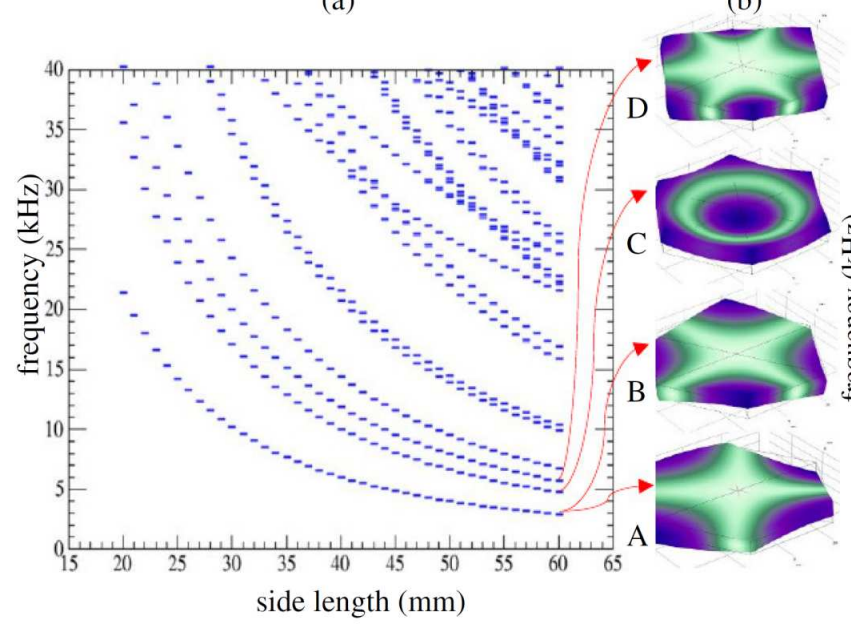

(c)

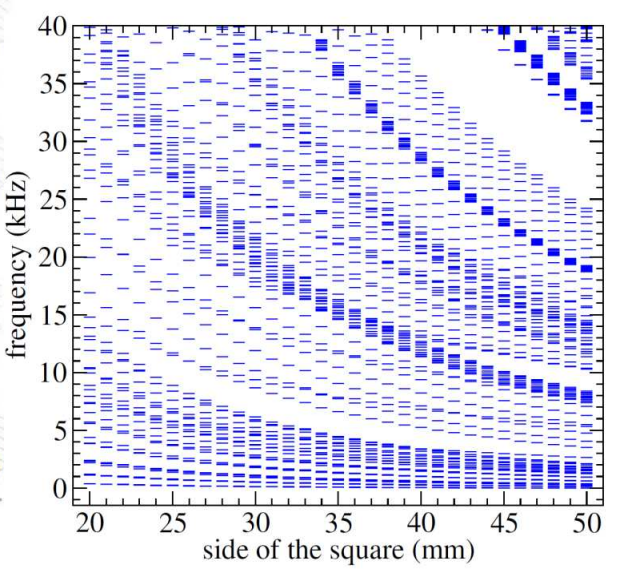

Fig. 2. (a) Frequency spectrum of a hexagonal plate as a function of its side length. (b) The fourth lowest wave amplitudes, two of them degenerate (A and B). Mode D will be used to build the elastic borazine meta-atoms. (c) Frequency spectrum of a finite phononic crystal composed of nine unit cells (see the text). 


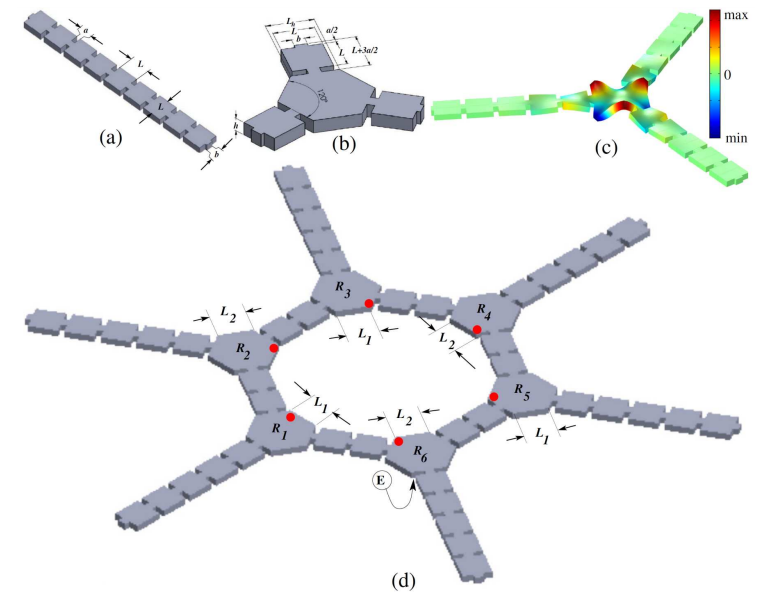

Fig. 3. Finite phononic crystal (FPC) composed of nine unit cells. Each unit cell is composed of a large square plate and two small rectangular plates. (b) The meta-atom is composed of a hexagonal plate and three connectors with one unit cell of the FPC. (c) Artificial elastic $\pi$-orbital, or elastic meta-orbital, associated with a localized wave amplitude. (d) The artificial elastic borazine molecule is composed of three meta-atoms with $L_{h}=L_{1}$ and three with $L_{h}=L_{2}$. Notice that FPCs with 4 unit cells were used to minimize border effects (one unit cell belongs to the meta-atom).

When a second resonator is placed close to the first one they weakly couple to each other through an elastic analog of tunneling [6]. Thus a tightbinding (TB) regime for elastic waves emerges [8]. This TB regime has been used to create 1D molecules that satisfy the quantum TB model $[7,8]$ and more recently the benzene $\pi$-orbitals were emulated [9]. We want to emphasize that the coupling between resonators used here is evanescent as the one in the dielectric resonators [10], in contrast to that encountered in microwave networks [27].

\section{Numerical simulations of the elastic borazine}

The artificial elastic borazine is shown in Fig. 3d. It is composed of six resonators, six connectors, and six terminators. The latter, composed of four unit cells of the FPC (one unit cell belongs to the metaatom), are used to avoid unwanted edge effects from the free surface. When all resonators are equal, artificial benzene orbitals emerge [9]. Here, to emulate the borazine orbitals, three resonators have length $L_{1}$ and the other three $L_{2}$ (see Fig. 3d). The resonant frequencies of both kinds of resonators are taken in the bandgap of the FPC. By changing the size $L_{2}$ of resonators $R_{2}, R_{4}$, and $R_{6}$ around the fixed size $L_{1}=30.4 \mathrm{~mm}$ of the other resonators, the artificial elastic borazine can be engineered. In Fig. 4 the normal-mode frequencies of the elastic molecule are shown as a function of $L_{2}$. Although

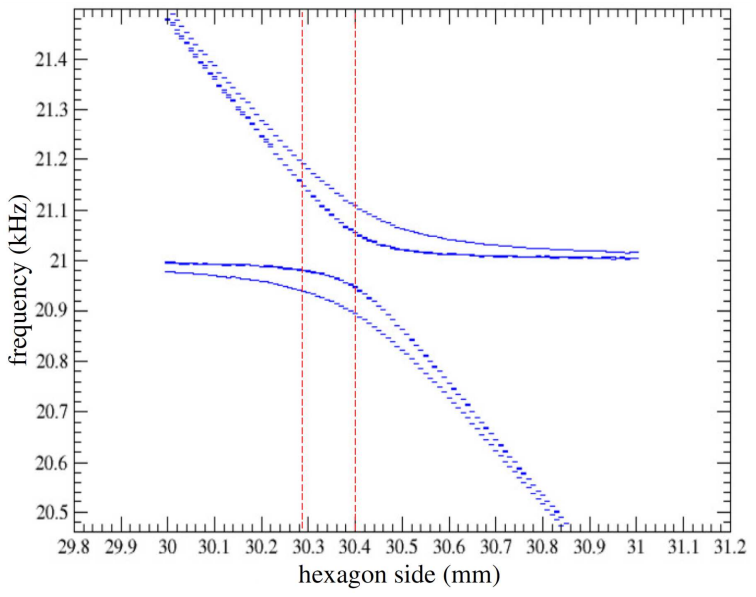

Fig. 4. Normal-mode frequencies associated with the artificial elastic aromatic molecules with six atoms. Right line: benzene. Left line: borazine. The inner curves correspond to degenerated eigenvalues; then six levels appear.

only four levels are visible, there are six normalmode frequencies, associated with each meta-orbital of the artificial molecule. The inner levels are doubly degenerated and the spectrum of the artificial elastic borazine comprises two singlets, at the border, and two inner doublets. When all resonators are equal, the frequency difference between the doublets is twice the frequency difference between doublet and singlet. This case corresponds to the artificial elastic benzene (vertical red line at the right) [9]. The case of borazine will be taken as the one in which the size of the resonators yields a frequency difference $\Delta$ between the doublets equal to 3.96 times the frequency difference $\delta$ between singlet and doublet, as happens in the real borazine [24]. In the artificial elastic borazine, the precision of the CNC machines only reaches an approximate value of 3.97 for this factor. This case is indicated by the red vertical line at the left in Fig. 4. The normal-mode wave amplitudes are given in Fig. 5. As it can be seen, the artificial orbitals present partial localization. This is in agreement with the results of [24].

\section{Tight-binding (TB) model for artificial borazine}

The spectrum of the artificial elastic borazine can be obtained with the TB formalism. The nearestneighbor Hamiltonian for the borazine, in terms of the meta-atomic orbitals $|i\rangle$ is given by

$$
H=\left(\begin{array}{cccccc}
f_{\mathrm{B}} & C & 0 & 0 & 0 & C \\
C & f_{\mathrm{N}} & C & 0 & 0 & 0 \\
0 & C & f_{\mathrm{B}} & C & 0 & 0 \\
0 & 0 & C & f_{\mathrm{N}} & C & 0 \\
0 & 0 & 0 & C & f_{\mathrm{B}} & C \\
C & 0 & 0 & 0 & C & f_{\mathrm{N}}
\end{array}\right)
$$




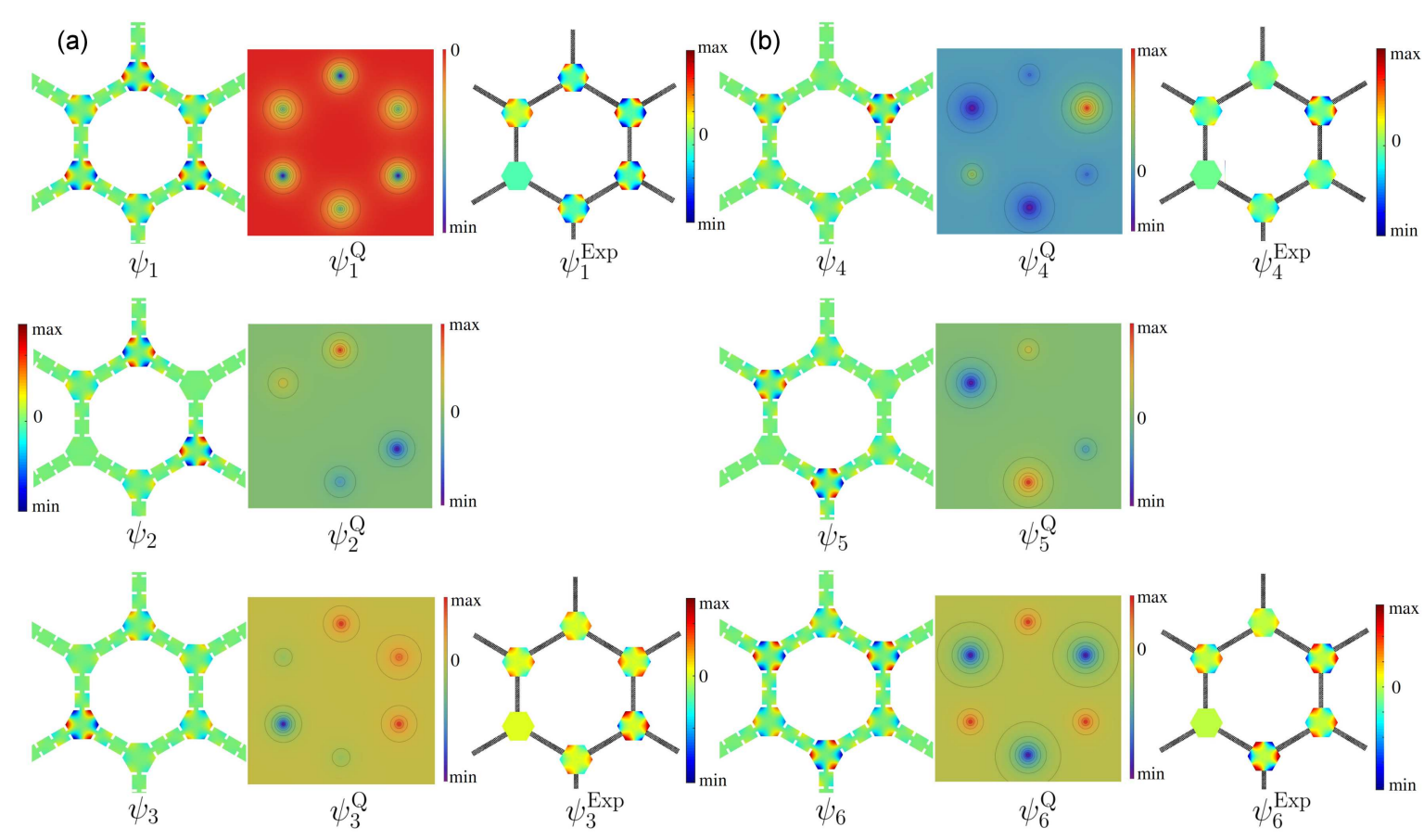

Fig. 5. (a and b) Artificial borazine orbitals versus borazine orbitals versus experimental orbitals, all in configuration space. Left column: numerical artificial borazine orbitals obtained with finite elements. Middle column: borazine quantum orbitals obtained with the tight-binding formalism [24]. Right column: experimental artificial borazine orbitals. Notice that there are many choices to take the orbitals corresponding to degenerate eigenvalues.

where $f_{\mathrm{B}}$ and $f_{\mathrm{N}}$ are the normal-mode frequencies of the boron and nitrogen meta-atoms. It is assumed a nearest-neighbor coupling $C$ between meta-orbitals. The artificial elastic molecular amplitude or meta-molecular orbital $\Psi$, in terms of the artificial meta-atom orbitals $|i\rangle$ will be

$$
|\Psi\rangle=\sum_{i} a_{i}|i\rangle
$$

The eigenvalues of the TB Hamiltonian, given in (1), are

$$
f_{1}=f_{c}-\sqrt{\delta^{2}+4 C_{2}}
$$

$$
\begin{aligned}
& f_{2,3}=f_{c}-\sqrt{\delta^{2}+C_{2}}, \\
& f_{4,5}=f_{c}+\sqrt{\delta^{2}+C_{2}}, \\
& f_{6}=f_{c}+\sqrt{\delta^{2}+4 C_{2}},
\end{aligned}
$$

where $f_{c}=\frac{1}{2}\left(f_{\mathrm{B}}+f_{\mathrm{N}}\right)$ and $\delta=\frac{1}{2}\left(f_{\mathrm{B}}-f_{\mathrm{N}}\right)$. This structure arises from the time-reversal symmetry and cyclicity of $H$ and agrees with that reported in [24]. The eigenfunctions (wave amplitudes) are given by

$$
\begin{aligned}
& \left|\Psi_{1}\right\rangle=A_{1}\left[-\left(\eta+\sqrt{1+\eta^{2}}\right)|1\rangle+|2\rangle-\left(\eta+\sqrt{1+\eta^{2}}\right)|3\rangle+|4\rangle-\left(\eta-\sqrt{1+\eta^{2}}\right)|5\rangle+|6\rangle\right] \\
& \left|\Psi_{2}\right\rangle=A_{2}\left[-\left(\eta+\sqrt{1+\eta^{2}}\right)|1\rangle+\left(\eta+\sqrt{1+\eta^{2}}\right)|3\rangle-|4\rangle+|6\rangle\right] \\
& \left|\Psi_{3}\right\rangle=A_{3}\left[-|1\rangle-\left(\eta-\sqrt{1+\eta^{2}}\right)|2\rangle+\left(\eta-\sqrt{1+\eta^{2}}\right)|4\rangle+|5\rangle\right] \\
& \left|\Psi_{4}\right\rangle=A_{4}\left[-\left(\eta-\sqrt{1+\eta^{2}}\right)|1\rangle+\left(\eta-\sqrt{1+\eta^{2}}\right)|3\rangle-|4\rangle+|6\rangle\right] \\
& \left|\Psi_{5}\right\rangle=A_{5}\left[-|1\rangle-\left(\eta+\sqrt{1+\eta^{2}}\right)|2\rangle-\left(\eta+\sqrt{1+\eta^{2}}\right)|4\rangle+|5\rangle\right], \\
& \left|\Psi_{6}\right\rangle=A_{6}\left[-\left(\eta-\sqrt{1+\eta^{2}}\right)|1\rangle+|2\rangle-\left(\eta-\sqrt{1+\eta^{2}}\right)|3\rangle+|4\rangle-\left(\eta-\sqrt{1+\eta^{2}}\right)|5\rangle+|6\rangle\right]
\end{aligned}
$$




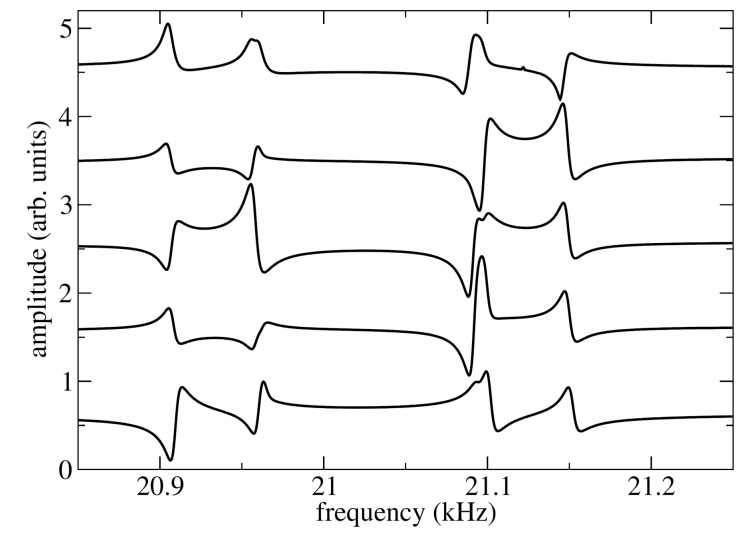

Fig. 6. Spectra of the artificial borazine orbitals measured in the red dots of Fig. 3. The curves from bottom to top correspond to measurements in resonators $R_{1}, R_{2}, R_{3}, R_{4}$, and $R_{5}$ of Fig. 3 .

Here $\eta=\frac{1}{2} \delta / c$ and $A_{i}, i=1, \ldots, 6$ are normalization constants. Notice that the eigenfunctions corresponding to degenerate orbitals $\left|\Psi_{2}\right\rangle$ and $\left|\Psi_{3}\right\rangle$, and those corresponding to $\left|\Psi_{4}\right\rangle$ and $\left|\Psi_{5}\right\rangle$, are not unique. The eigenfunctions, in the configuration space, are plotted in Fig. 5. These amplitudes agree with the quantum molecular orbitals $\phi^{\mathrm{Q}}$ of [24], plotted in the middle columns of Fig. $5 \mathrm{a}$ and $\mathrm{b}$ for completeness, also supposing the nearest-neighbor coupling. Notice that the artificial elastic molecular orbitals differ from the quantum molecular orbitals only in the site orbitals used. In addition, the degenerate waves can always be chosen as real, in contrast to symmetry-adapted bases.

\section{Experimental realization of borazine}

To confirm the predictions of the numerical design of the artificial elastic borazine, the structure shown in Fig. 3 was experimentally characterized. For this purpose, a variation of the resonant ultrasound spectroscopy technique was used. This technique is described in detail in [28-30] so it will not be discussed here. In Fig. 3 the resonators of the nitrogen and boron meta-atoms are labeled as $R_{1}$ and $R_{2}$, respectively. Below the resonator $R_{1}$, an electromagnetic-acoustic transducer (EMAT) was placed to produce out-of-plane vibrations without physical contact. This exciter is indicated by an "E" in the same figure. The response of the elastic system was recorded in the other resonators with an EMAT detector. The detector was placed in the equivalent positions of each resonator, indicated by the red dots in Fig. 3. The measured response at these points is plotted in Fig. 6. Each resonance shows an asymmetric line due to the Fano effect [31]. As it can be seen, four resonances appear although, in the measurement, in the first and third resonators $\left(R_{1}\right.$ and $\left.R_{3}\right)$ a slight distortion of the third resonance appears, which evidences the existence of the corresponding doublet. The separation between the singlet and the consecutive doublet is almost a quarter the separation between the two consecutive doublets, which confirms the theoretical predictions. Four orbitals can be obtained taking the measurements within each resonator of [9] with the amplitudes and phases shown in Fig. 6. In the right column of Fig. 5, four measured orbitals are given. As it can be seen, the experiments have a very good agreement with the numerical simulations and with the theoretical predictions of the tight-binding model. More experiments using clamps, like those performed in [9], are needed to obtain the missing orbitals.

\section{Conclusions}

We have designed and constructed an artificial elastic borazine molecule. The artificial molecule is composed of six meta-atoms composed of elastic resonators and finite phononic crystals. The meta-atoms normal modes have localized wave amplitudes or atomic meta-orbitals, that connect weakly to each other through evanescent Bloch waves. When two kinds of meta-atoms are connected forming a hexagonal ring, the artificial elastic borazine emerges and its artificial molecular orbitals agree with those of the real molecular orbitals. The artificial elastic borazine molecules were engineered using the finite element method and experiments performed with resonant ultrasound spectroscopy corroborate the findings. A very good agreement with the simplest tight-binding formalism was obtained.

\section{Acknowledgments}

This work it was supported by DGAPAUNAM and by CONACYT under projects PAPIIT IN111021 and CB2016/284096. The authors collaborated under the "Waves and Metamaterials Group" initiative. The authors acknowledge the kind hospitality of Centro Internacional de Ciencias A.C. for its facilities for group meetings and gatherings hosted there and for space to locate the Laboratory of Waves and Metamaterials, where the experiments were performed. G.B. received funding from CONACYT under project A1-S-33920.

\section{References}

[1] K.H. Matlack, M. Serra-Garcia, A. Palermo, S.D. Huber, C. Daraio, Nat. Mater. 17, 323 (2018).

[2] Y. Jin, D. Torrent, B. Djafari-Rouhani, Phys. Rev. B 98, 054307 (2018).

[3] D. Torrent, J. Sánchez-Dehesa, Phys. Rev. Lett. 108, 174301 (2012).

[4] M. Dubois, C. Shi, X. Zhu, Y. Wang, X. Zhang, Nat. Commun. 8, 14871 (2017). 
[5] F.D. Philippe, T. W. Murray, C. Prada, Sci. Rep. 5, 11112 (2015).

[6] F. Ramírez-Ramírez, R. A. MéndezSánchez, G. Báez, A. Morales, L. Gutiérrez, J. Flores, in: 2018 Progress in Electromagnetics Research Symposium (PIERS), Toyama (Japan), 2018, p. 410.

[7] F. Ramírez-Ramírez, E. Flores-Olmedo, G. Báez, E. Sadurní, R.A. MéndezSánchez, Sci. Rep. 10, 10229 (2020).

[8] J.A. López-Toledo, G. Báez, R.A. MéndezSánchez, Mech. Adv. Mater. Struct. 0, 1 (2021).

[9] A.M. Martínez-Argüello, M.P. ToledanoMarino, A.E. Terán-Juárez, E. FloresOlmedo, G. Báez, E. Sadurní, R.A. Méndez-Sánchez, arXiv:2108.12027, 2021.

[10] J.A. Franco-Villafaẽ, E. Sadurní, S. Barkhofen, U. Kuhl, F. Mortessagne, T.H. Seligman, Phys. Rev. Lett. 111, 170405 (2013).

[11] A. Yariv, Yong Xu, R.K. Lee, A. Scherer, Opt. Lett. 24, 711 (1999).

[12] E. Ozbay, M. Bayindir, I. Bulu, E. Cubukcu, IEEE J. Quantum Electron. 38, 837 (2002).

[13] J. M. Escalante, A. Martínez, V. Laude, J. Phys. D Appl. Phys. 46, 475301 (2013).

[14] D. Reyes, D. Martínez, M. Mayorga, H. Heo, E. Walker, A. Neogi, Front. Mech. Eng. 6, 96 (2020).

[15] Ting-Ting Wang, S. Bargiel, F. Lardet Vieudrin, Yan-Feng Wang, Yue-Sheng Wang, V. Laude, Phys. Rev. Appl. 13, 014022 (2020).

[16] M. S. Kushwaha, P. Halevi, L. Dobrzynski, B. Djafari-Rouhani, Phys. Rev. Lett. 71, 2022 (1993).

[17] M. Sigalas, E.N. Economou, Solid State Commun. 86, 141 (1993).

[18] A. Morales, J. Flores, L. Gutiérrez, R.A. Méndez-Sánchez, J. Acoust. Soc. Am. 112, 1961 (2002).
[19] A. Díaz de Anda, A. Pimentel, J. Flores, A. Morales, L. Gutiíerrez, R. MéndezSánchez, J. Acoust. Soc. Am. 117, 2814 (2005).

[20] Yu Dianlong, Liu Yaozong, Wang Gang, Zhao Honggang, Qiu Jing, J. Appl. Phys. 100, 124901 (2006).

[21] Liu Liao, H.I. Mahmoud, J. of Appl. Mech. 79, 011003 (2012).

[22] Y.Q. Guo, D.N. Fang, J. Appl. Mech. 81, 011009 (2014).

[23] S.F. Parker, RSC Adv. 8, 23875 (2018).

[24] Y. Hernández-Espinosa, R.A. MéndezSánchez, E. Sadurní, J. Phys. B At. Mol. Opt. Phys. 53(10), 105101 (2020).

[25] A. Arreola-Lucas, G. Báez, R.A. MéndezSánchez, A. Climente, F. Cervera, J. Sánchez-Dehesa, in: 2018 Progress in Electromagnetics Research Symposium (PIERS), Toyama (Japan) 2018, p. 2399.

[26] A. Arreola-Lucas, G. Báez, F. Cervera, A. Climente, R.A. Méndez-Sánchez, J. Sánchez-Dehesa, Sci. Rep. 9, 1860 (2019).

[27] B. Dietz, V. Yunko, M. Białous, S. Bauch, M. Ławniczak, L. Sirko, Phys. Rev. E 95, 052202 (2017).

[28] B. Manzanares-Martínez, J. Flores, L. Gutiérrez, R.A. Méndez-Sánchez, G. Monsivais, A. Morales, F. RamosMendieta. J. Sound Vib. 329, 5105 (2010).

[29] J.A. Franco-Villafañe, E. Flores-Olmedo, G. Báez, O. Gandarilla-Carrillo, R.A. Méndez-Sánchez, Eur. J. Phys. 33, 1761 (2012).

[30] E. Flores-Olmedo, A.M. MartínezArgüello, M. Martínez-Mares, G. Báez, J.A. Franco-Villafañe, R.A. MéndezSánchez, Sci. Rep. 6, 25157 (2016).

[31] A.M. Martínez-Argüello, M. MartínezMares, M. Cobián-Suárez, G. Báez, R.A. Méndez-Sánchez, EPL (Europhys. Lett.) 110, 54003 (2015). 